\title{
Deep image mining for convolution neural network
}

\author{
Dhamea A. Jasm ${ }^{1}$, Murtadha M Hamad ${ }^{2}$, Azmi Tawfek Hussein Alrawi ${ }^{3}$ \\ Department of Computer Sciences, Collage of computer sciences and Information Technology, Iraq
}

\begin{tabular}{l}
\hline Article Info \\
\hline Article history: \\
Received Feb 6, 2020 \\
Revised Apr 8, 2020 \\
Accepted Apr 22, 2020 \\
\hline
\end{tabular}

Keywords:

$\mathrm{CNN}$

DCNN

Images classification

\begin{abstract}
Image mining is the method of searching and discovering valuable information and knowledge from a huge image dataset. Image mining is based on data mining, digital image processing, machine learning, image retrieval, and artificial intelligence. Image mining handled with the hidden information extraction, an association of image data and additional pattern which are not clearly visible in the image. Choosing the proper objects or the feature of the image to be suitable for image mining process is the main challenge would face the programmer. The process includes fine out the most efficient routes at a shorter time and saving the users effort. The main objective of this paper is to design and implement the image classification system with a higher performance, where a CIFAR-10 data set is used to train and testing classification models using CNN. A convolutional neural network is trustworthy, and it could lead to high-quality results. The high accuracy of $98 \%$ has been obtained using deep convolutional neural network (DCNN).
\end{abstract}

Copyright $\odot 2020$ Institute of Advanced Engineering and Science. All rights reserved.

\section{Corresponding Author:}

Dhamea A. Jasm,

Department of Computer Sciences and Information Technology,

University of Anbar, Al-Anbar, Iraq.

Email: dhameaanwar@gmail.com

\section{INTRODUCTION}

Deep learning can be defined as a set of processes by which the patterns of the data can be automatically excluded. After which the revealed data is used for further data prediction or being utilized to estimate the correct decision at uncertain conditions [1]. The DL is identified from the data, and the decision processed with a limit of human's interventions [2]. Firstly, the analysis of the training data needs to be figured out before making any prediction into the new data [3]. The effectiveness of the deep convolutional neural network (DCNN), which is a special type of artificial neural network (ANN), needs to be tested before being conducted in the photo recognition task $[4,5]$. Currently, image mining (IM) grabs significant attention by the researchers due to the unlimited available images whether it is stored or displayed on the internet. A simple way to classify the images is by dividing the images into groups containing classes, extracting the important features from the huge number of images during a short time [6]. The classification process of the images is a sophisticated process due to unstructured image data which is also associated with the noise $[7,8]$. The DCNN is very efficient and has been used effectively in a large-scale object recognition of the images. In this paper, an image database was used in groups of different patterns, colors, and shapes to investigate the efficiency of the algorithm in classifying any image related to any of those groups inside the database, and presenting the most important layers in the algorithm Deep Convolutional Neural Network (DCNN).

Senapati [9], proposed utilizing color fundus images to identify the bright lesions or exudates using a computer associated with a screening system. The suspicious regions of the bright lesions were firstly detected by the screening system then the interested region under investigation was characterized by the texture feature extraction method. The last stage was performed using the support vector machine classifier to classify the images into normal and abnormal images. S. Kumar et al. [10], applied a hybrid approach. To extract the features from the images the approach conducted the discrete wavelet transform (DWT) for 
that purpose, while the Genetic algorithm is used to diminish the number of features. Finally, the support vector machine (SVM) is used to classify the brain tumor. In comparison with the other reported techniques, an improvement in the accuracy and a minimizing in the RMS error (a similar context Linear accuracy was $80 \%-90 \%$ ) is recorded via using the hybrid approach.

Vision-based vehicle classification has been investigated by R. Durratun Safiyah, et al. [11], The evaluation has been conducted using Convolutional Neural Network (CNN) models. The CNN models are simple CNN, and pre-trained CNN models (AlexNet and GoogleNet). The optimum results are achieved by AlexNet using dataset includes 7000 images. N. N. A. A. Hamid, R. A. Razali et al. [12], compared three different models which are Conventional Convolutional Neural Network (CNN), Bag of Features (BoF) and Alexnet for fruiter cognition aiming to achieve a lower human interference in the fruit harvesting, in addition, to reduce the cost and the time of the harvesting process. B. B. Traore, B. Kamsu-Foguem, et al. [13], conducted the DCNN recognition approach to classify the microscopic images which include cholera or malaria. W. Lumchanow and S. Udomsiri [14], This paper presents image classification algorithms to improve the learning rate and to compare the classification efficiency. Using a convolutional neural network $(\mathrm{CNN})$ for feature extraction and method to find appropriate $\mathrm{k}$ for the k-nearest neighbor (KNN). Medical datasets were used in the experiments to classify Plasmodium Vivax and Plasmodium Falciparum.

An evaluation study on leaf recognition carried out by N. F. Sahidan et al. [15], by adopting both $(\mathrm{CNN})$ and Bag of Features $(\mathrm{BoF})$ and getting the benefit of the available public database called Folio dataset. CNN exhibited a powerful feature performance in computer vision. H. Sofian, J. T. C. Ming, S. Muhammad et al. [16], for classification of Coronary artery, the researchers conducted the available networks using Cartesian Coordinates and polar reconstructed coordinate images for a deep understanding of the ultrasound images. For a real-time detection of cancer cells, A. A. Abdullah, et al., [17], developed a MATLAB based CNN algorithm for auto-detection. The proposed solution based on segmenting the nuclear of the cancerous cell. The detection of the algorithm was of accuracy about $88 \%$.

The related works are shown many researchers have used various algorithms to classify images and got more knowledge to take high accuracy decisions. CNN was the more algorithm used in the classification of the image in modern studies. But, still a lot of works to images classification in the huge datasets. Therefore, in this research employ the CNN algorithm for classification huge dataset which are CIFAR-10 [18], and compare the result with some previous work that has displayed in related works to determine which algorithm the best.

\section{MATERIALS AND METHOD}

\subsection{Convolutional neural network $(\mathrm{CNN})$}

$\mathrm{CNN}$ is a class of neural network models that includes huge network layers such as an input image [19], convolution layer, average pooling, max pooling and rectified linear unit, etc. The number of layers controls the size of the input images. However, some of those layers might be used in the network. Better learning can be achieved from a considerable large network size which can allow for a self-learn and self-organize [20-22]. However, the disadvantage of the large and more depth network is the increment the computational time. The current work is an attempt to select the proper parameters which can allow using a minimum number of layers to obtain maximum output from the network [7]. The CNN does need to involve other methods for pre-processing or handcrafted feature extraction. The hierarchical manner is utilized to extract the features. The process involves depicting and classifying the objects of the input image. For higher classification performance could be achieved by adjusting the parameters $[13,23,24]$. Figure 1 describes the different CNN units.

Convolution performed in each layer consists of four steps as follows:

Step 1: Placing a filter on the position of the input image

Step 2: Calculating the position of the center of datasets from (1):

$$
(I \times h)[i, j]=\sum_{u, v} h(u, v) \times I(i+u, j+v)
$$

Where $I$ refer to the input matrix, whose size is equal to that of the filter;

$h$ is a medium matrix;

$I$ and $j$ is the central position of matrix considering positions;

$U$ and $\mathrm{v}$ are positions of the medium used in the calculation.

Step 3: Moving filters to different positions on the input image pixel to pixel until the last one

Step 4: Repeating Step 2 to Step 3.

Follow convolution layer of the Rectified Linear Unit (ReLu) proposed setting the value of zero for each value less than zero for any element [25]. The process leads to reduce the abundance of the data and not undamaged the important features. However, the output layer has the same size as that of the previous layer. 
To reduce the size of the layer, Max pooling was applied. Over the window, a maximum value has been considered and it replaced the window. The size is lower for the output layer compared to the previous layer [26]. From the process of convolution algorithm, the more convolutional layers required more time for calculation. In this regard, max pooling is an algorithm used to reduce calculation problems and increase calculation speed [8]. Upon completion of the feature extraction process using filters and max pooling $[27,28]$, the output will be in the form of a dataset that must be pre-processing through re-arrangement to be in the form of one-dimension vector. From the CNN structure, the neurons of the previous layer i.e. maxpooling layer, a fully connected layer (FC), are connected to each neuron of that layer. The output would be the number of generated classes on that classification for that layer $[29,13]$.

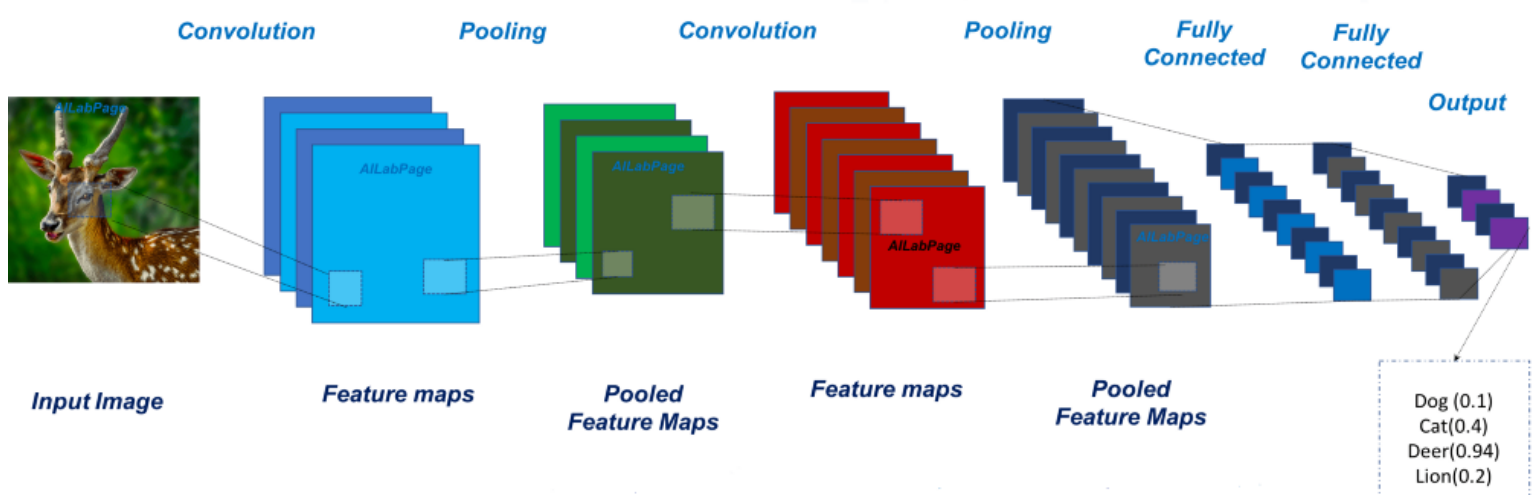

Figure 1. The general CNN architecture

\subsection{Dataset}

The python has been used in the experiments of the current research. The dataset of the CIFAR-10 is containing 10 classes of $60,000,32 \times 32$ image's color. Each class includes 6000 images. Furthermore, the number of training images inside the system is 50,000 in addition to 10,000 test images. Python is used for the programming language of machine learning conducted in this research. Figure 2 illustrates a dataset image contains ten classes.

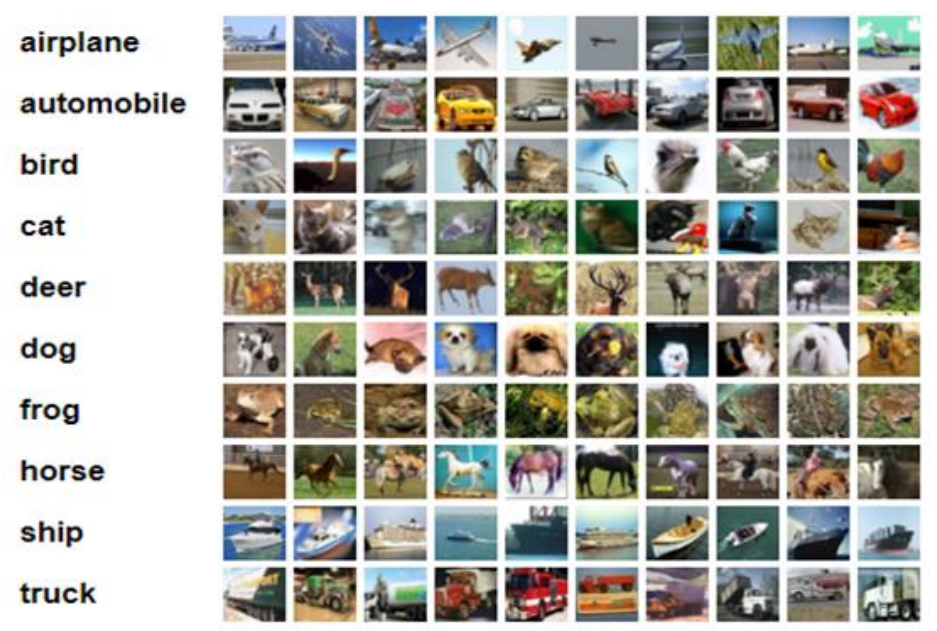

Figure 2. sample images from the dataset for ten classes

\section{RESULTS AND ANALYSIS}

$\mathrm{CNN}$ is structured of three hidden layers which are two convolution layers (each convolution layer includes the same architecture) followed by a ReLU element-wise nonlinearity and MaxPooling layer. The convergence of the error rate during the learning process decides the criteria by which the number of 
convolution layers is being chosen. Here, to generate the experiment, 2 or 3 iterations have been considered (via enlarged the number of convolution layers) to calculate the converge. Figure 3 and Table 1 summarizes the CNN training architecture for this research. The CNN training algorithm is conducted with a Stochastic gradient descent via choosing a random data contains small and equal batches of each iterative learning phase. The challenge of proving that it is a technique that is best to distinguish objects in the image. Accuracy and Loss from CNN as shown in Figure 4.

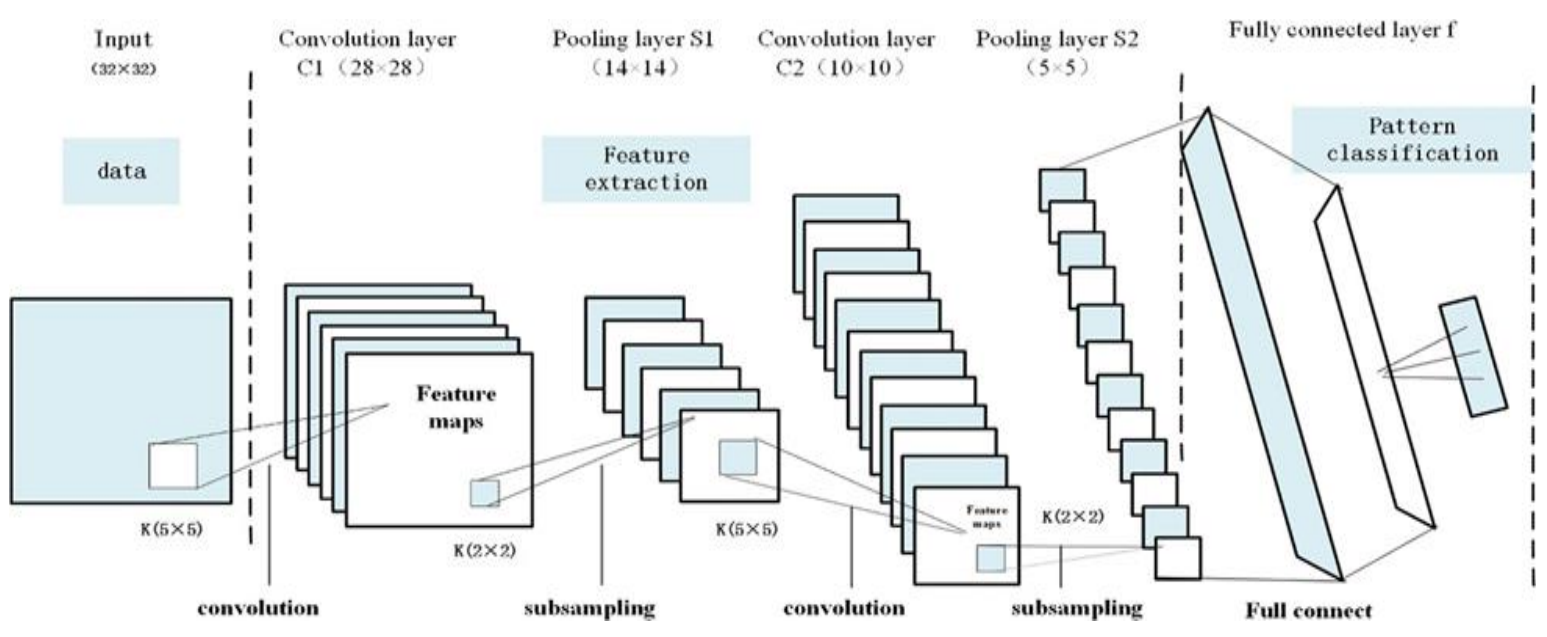

Figure 3. The choosing CNN Training architecture

Table 1. The ultimate steps of the CNN training model

\begin{tabular}{cccccc}
\hline Parameters of the model & Training step & The accuracy & Val_accuracy & Loss & Val_loss \\
\hline Values & 383 (last step) & 0.9765 & $0.98 \%$ & 0.5670 & $0.58 \%$ \\
\hline
\end{tabular}
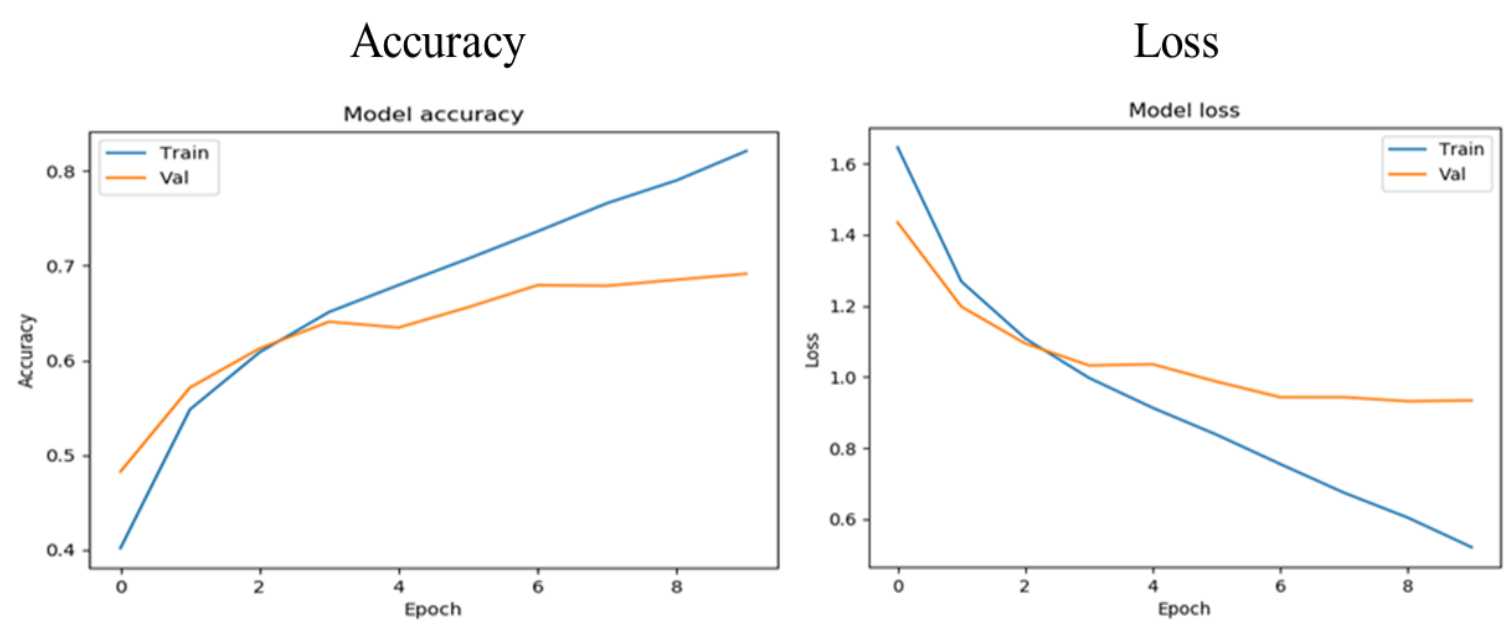

Figure 4. Accuracy and Loss from CNN

In this research of proving that it is the best technique Convolution Neural Network (CNN) to classify images when there is always a thousand pictures and the ability to predict the type of image, by taking 1 number of features extracted such of eye, nose, and mouth from the image, it has proven the ability to accurately classify $98 \%$ with hundreds of thousands of images In previous studies that relied on a few pictures as mentioned in Table 2. 
Table 2. Methods used in the previous related works with the proposed techniques

\begin{tabular}{|c|c|c|c|c|c|}
\hline Reference & Author & Years & Techniques & Number of datasets & Accuracy \\
\hline [9] & R. K. Senapati & 2016 & Support vector machine (SVM) & 1289 image & $96 \%$ \\
\hline [10] & S. Kumar et al. & 2017 & hybrid approach:(DWT) \& (SVM) & 145 image & $85 \%$ \\
\hline [11] & R. Durratun Safiyah & 2018 & $\begin{array}{l}\text { Convolutional Neural Network } \\
\text { (CNN). }\end{array}$ & 7000 image & $65 \%$ \\
\hline [12] & N. N. A. A. Hamid et al. & 2018 & Comparing (BoG), (CNN), Alexent & 1200 image & $\begin{array}{c}\text { BoG }=98 \% \\
\text { Alexent }=95 \% \\
\text { CNN }=99 \%\end{array}$ \\
\hline [13] & B. B. Traore et al. & 2018 & $\begin{array}{l}\text { Convolutional Neural Network } \\
\text { (CNN). }\end{array}$ & 240 image & $94 \%$ \\
\hline [14] & W. Lumchanow et al. & 2019 & Hybrid approach:(KNN) \& (CNN). & 940 image & $89 \%$ \\
\hline [15] & N. F. Sahidan et al. & 2019 & Hybrid approach:( BoG) \& (CNN). & 640 image & $82 \%$ \\
\hline [16] & H. Sofian et al. & 2019 & convolutional neural network (CNN). & 2175 image & $85 \%$ \\
\hline [17] & A. A. Abdullah et al. & 2019 & $\begin{array}{c}\text { cellular neural } \\
\text { network }\end{array}$ & 1500 image & $88 \%$ \\
\hline & Current Study & 2020 & Convolution neural network (CNN) & 60000 image & $98 \%$ \\
\hline
\end{tabular}

\section{CONCLUSION}

The current work pointed out the necessity of image mining due to the growth of the image datasets. Deep Convolution Neural Network has been operated for image classification. The image classification recognized the image with different shapes, colors, and texture to support the decision of making further processing. The CNN architecture achieved an optimum classification result with an accuracy of $98 \%$, with 60000 images. The robustness of the proposed system can be by using a large database. and deep reading in the researchers that used the techniques of image mining to classification. However, a further experiment on other datasets is necessary to be carried out using the learning machine and deep learning techniques. To enlarge the efficiency of the algorithm, it is important to estimate the number of hidden layers and their chronological order. A global Webs are considered as an image database containing an enormous number of images that can supply an unlimited amount of information, proposing suitable indexing and retrieving knowledge from images.

\section{REFERENCES}

[1] N. E. Binti Md Isa, et al., "Motor imagery classification in brain computer interface (BCI) based on EEG signal by using machine learning technique," Bull. Electr. Eng. Informatics (BEEI), vol. 8, no. 1, pp. 269-275, 2019.

[2] M. Armanur Rahman et al., "A survey of machine learning techniques for self-tuning hadoop performance," Int. J. Electr. Comput. Eng (IJECE)., vol. 8, no. 3, pp. 1854-1862, 2018.

[3] P. Patel and A. Thakkar, "The upsurge of deep learning for computer vision applications," Int. J. Electr. Comput. Eng (IJECE)., vol. 10, no. 1, pp. 538-548, 2020.

[4] A. A. M. Al-Saffar, et al., "Review of deep convolution neural network in image classification," Proceeding - 2017 Int. Conf. Radar, Antenna, Microwave, Electron. Telecommun. ICRAMET 2017, vol. 2018-Janua, pp. 26-31, 2017, doi: 10.1109/ICRAMET.2017.

[5] M. S. Fairuz, et al.,"Pre-trained based CNN model to identify finger vein," Bull. Electr. Eng. Informatics (BEEI), vol. 8, no. 3, pp. 855-862, 2019.

[6] N. A. Rahmad, et al., "Recognition of badminton action using convolutional neural network," Indones. J. Electr. Eng. Informatics, vol. 7, no. 4, pp. 750-756, 2019.

[7] S. Aich, et al., "Convolutional neural network-based model for web-based text classification," Int. J. Electr. Comput. Eng (IJECE)., vol. 9, no. 6, pp. 5185-5191, 2019.

[8] A. Setyono and D. R. I. M. Setiadi, "Image watermarking using discrete wavelet-tchebichef transform," Indones. J. Electr. Eng. Comput. Sci (IJEECS)., vol. 16, no. 3, pp. 1416-1423, 2019.

[9] R. K. Senapati, "Bright Lesion Detection in Color Fundus Images Based on Texture Features," Bull. Electr. Eng. Informatics (BEEI), vol. 5, no. 1, pp. 92-100, 2016.

[10] S. Kumar, et al., "Classification of Brain MRI Tumor Images: A Hybrid Approach," Procedia Comput. Sci., vol. 122, pp. 510-517, 2017.

[11] R. Durratun Safiyah, et al., "Performance Evaluation for Vision-Based Vehicle Classification Using Convolutional Neural Network," Int. J. Eng. Technol., vol. 7, no. 3.15, p. 86, 2018.

[12] N. N. A. A. Hamid, et al., "Comparing bags of features, conventional convolutional neural network and alexnet for fruit recognition," Indones. J. Electr. Eng. Comput. Sci (IJEECS)., vol. 14, no. 1, pp. 333-339, 2019.

[13] B. B. Traore, et al., "Deep convolution neural network for image recognition," Ecol. Inform., vol. 48, pp. 257-268, 2018.

[14] W. Lumchanow and S. Udomsiri, "Image classification of malaria using hybrid algorithms: convolutional neural network and method to find appropriate $\mathrm{K}$ for K-Nearest neighbor," Indones. J. Electr. Eng. Comput. Sci (IJEECS)., vol. 16, no. 1, pp. 382-388, 2019. 
[15] N. F. Sahidan, et al., "Evaluation of basic convolutional neural network and bag of features for leaf recognition," Indones. J. Electr. Eng. Comput. Sci (IJEECS)., vol. 14, no. 1, pp. 327-332, 2019.

[16] H. Sofian, et al., "Calcification detection using convolutional neural network architectures in intravascular ultrasound images," Indones. J. Electr. Eng. Comput. Sci (IJEECS)., vol. 17, no. 3, pp. 1313-1321, 2019.

[17] A. A. Abdullah, et al., "Cervical cancer detection method using an improved cellular neural network (CNN) algorithm," Indones. J. Electr. Eng. Comput. Sci (IJEECS)., vol. 14, no. 1, pp. 210-218, 2019.

[18] G. H. Krizhevsky, et al., "CIFAR-10 and CIFAR-100 datasets," 2009.

[19] Z. Faisal and N. K. El Abbadi, "Detection and recognition of brain tumor based on DWT, PCA and ANN," Indones. J. Electr. Eng. Comput. Sci (IJEECS)., vol. 18, no. 1, pp. 56-63, 2019.

[20] U. R. Acharya, et al., "Automated detection of arrhythmias using different intervals of tachycardia ECG segments with convolutional neural network," Inf. Sci. (Ny)., vol. 405, pp. 81-90, 2017.

[21] M. M. R. Krishnan and O. Faust, "Automated glaucoma detection using hybrid feature extraction in retinal fundus images," J. Mech. Med. Biol., vol. 13, no. 1, pp. 1-21, 2013.

[22] J. H. Tan et al., "Age-related Macular Degeneration detection using deep convolutional neural network," Futur. Gener. Comput. Syst., vol. 87, pp. 127-135, 2018.

[23] U. Raghavendra, et al., "Deep convolution neural network for accurate diagnosis of glaucoma using digital fundus images," Inf. Sci. (Ny)., vol. 441, pp. 41-49, 2018.

[24] J. Lee et al., "Deep Learning in Medical Imaging Gen," vol. 18, no. 4, pp. 570-584, 2017.

[25] S. Shindo, et al., "An optimization of facial feature point detection program by using several types of convolutional neural network," Indones. J. Electr. Eng. Comput. Sci (IJEECS)., vol. 16, no. 2, pp. 827-834, 2019.

[26] V. Suárez-Paniagua and I. Segura-Bedmar, "Evaluation of pooling operations in convolutional architectures for drug-drug interaction extraction," BMC Bioinformatics, vol. 19, pp. 92-101, 2018.

[27] M. Z. Osman, et al., "A multi-color based features from facial images for automatic ethnicity identification model," Indones. J. Electr. Eng. Comput. Sci (IJEECS)., vol. 18, no. 3, pp. 1383-1390, 2020.

[28] R. Rassetiadi and Suharjito, "Foreign exchange prediction based on indices and commodities price using convolutional neural network," Indones. J. Electr. Eng. Comput. Sci (IJEECS)., vol. 18, no. 1, pp. 494-501, 2019.

[29] H. F. Pardede, et al., "Convolutional Neural Network and Feature Transformation for Distant Speech Recognition," Int. J. Electr. Comput. Eng (IJECE)., vol. 8, no. 6, p. 5381, 2018. 